\title{
Resistivity of Dilute 2D Electrons in an Undoped GaAs Heterostructure
}

\author{
M. P. Lilly, ${ }^{1}$ J. L. Reno, ${ }^{1}$ J. A. Simmons, ${ }^{1}$ I. B. Spielman, ${ }^{2}$ J. P. Eisenstein, ${ }^{2}$ L. N. Pfeiffer, ${ }^{3}$ K. W. West, ${ }^{3}$ \\ E. H. Hwang, ${ }^{4}$ and S. Das Sarma ${ }^{4}$ \\ ${ }^{1}$ Sandia National Laboratories, Albuquerque, New Mexico 87185 \\ ${ }^{2}$ California Institute of Technology, Pasadena, California 91125 \\ ${ }^{3}$ Bell Laboratories, Lucent Technologies, Murray Hill, New Jersey 07974 \\ ${ }^{4}$ Condensed Matter Theory Center, University of Maryland, College Park, Maryland 20742
}

(Received 8 October 2002; published 7 February 2003)

\begin{abstract}
We report resistivity measurements from 0.03 to $10 \mathrm{~K}$ in a dilute high mobility $2 \mathrm{D}$ electron system. Using an undoped GaAs/AlGaAs heterojunction in a gated field-effect transistor geometry, a wide range of densities, $0.16 \times 10^{10}$ to $7.5 \times 10^{10} \mathrm{~cm}^{-2}$, are explored. For high densities, the results are quantitatively shown to be due to scattering by acoustic phonons and impurities. In an intermediate range of densities, a peak in the resistivity is observed for temperatures below $1 \mathrm{~K}$. This nonmonotonic resistivity can be understood by considering the known scattering mechanisms of phonons, bulk, and interface ionized impurities. Still lower densities appear insulating to the lowest temperature measured.
\end{abstract}

DOI: $10.1103 /$ PhysRevLett.90.056806

PACS numbers: 71.30.+h, 73.40.-c, 73.50.Bk

The resistivity of $2 \mathrm{D}$ electrons at zero magnetic field has been used to probe scattering and quantum processes for many years. Interest in the conducting behavior of $2 \mathrm{D}$ electrons and holes was further heightened in 1994 when Kravchenko and co-workers discovered an apparent metal-insulator transition of 2D electrons in Si metaloxide-semiconductor field-effect transistors (MOSFETs) [1]. While 2D systems are expected to exhibit insulating behavior (weak or strong localization) at sufficiently low temperatures and/or densities, the metallic side of the transition is more puzzling. A true metal conducts at $T=0$, however here we use the term "metallic" as referring to $d \rho / d T \geq 0$ at low temperature, where $\rho$ is the resistivity. Metallic behavior has been seen in a number of different material systems, including electrons in $\mathrm{Si}$ MOSFETs [1], holes in GaAs [2-4], and electrons in GaAs $[5,6]$ provided the carrier density is reasonably low (but not so low that the system is in the insulating phase) and the mobility relatively high. While all of these experimental systems exhibit metallic behavior in certain regimes of density $(n)$ and temperature, the quantitative behavior varies widely. As the temperature is lowered, $\rho$ can decrease by a factor of 10 in Si MOSFETs [1] compared to only a few percent for electrons in GaAs [5]. The drop in $\rho$ is typically observed when the parameter $r_{s}$ (ratio of Coulomb to kinetic energy, $\sim n^{-1 / 2}$ ) is much larger than 1, suggesting that electron-electron interactions may be important. The issue here is understanding the physical mechanisms underlying the metallic behavior and elucidating the roles of disorder and interaction.

In this Letter, we present the temperature dependence of the resistivity of a dilute 2D electron gas (2DEG) in GaAs. Electron-electron interactions can be extremely important in this system at low temperatures due to the low amount of disorder in GaAs heterostructures. In the sample discussed here, the density can be continuously tuned from $0.16 \times 10^{10} \mathrm{~cm}^{-2} \quad\left(r_{s}=13.7\right)$ to $7.5 \times$ $10^{10} \mathrm{~cm}^{-2}\left(r_{s}=2.0\right)$. With the ability to achieve very high $r_{s}$ in low disorder samples, these resistivity measurements provide a test of the importance of Coulomb interactions in the metallic regime. In particular, the $r_{s}$ ( $\gtrsim 10$ ) values of our 2 DEGs are very comparable to those in the best $\mathrm{Si}$ MOSFETs, whereas the disorder in our sample (as measured by $k_{F} \ell, k_{F}$ is the Fermi wave vector and $\ell$ the mean-free path) is more than 2 orders of magnitude lower. Earlier experimental studies of the metallic behavior in 2D electrons in GaAs systems were restricted to higher densities and lower mobilities, and the drop in resistivity was much weaker than the $20 \%$ drop observed here. Finally, the wide range of $n$ and $T$ reported here allows several scattering regimes to be identified.

We find that our measured temperature dependence of the resistivity can be qualitatively well understood within the framework of Fermi liquid theory for all but the lowest densities. At high density, the resistivity is linear with temperature, and the slope agrees both with previous measurements $[7,8]$ and established theory for acoustic phonon scattering [9]. For intermediate densities, a low temperature peak in the resistance is observed. The nonmonotonic resistivity agrees qualitatively with the predicted temperature dependence of ionized impurity scattering (screening at low $T$ and the transition from a degenerate Fermi gas to a classical system at high $T$ ).

The sample used in this study is a high mobility $2 \mathrm{DEG}$ confined at the interface of a GaAs/ $/ \mathrm{Al}_{0.3} \mathrm{Ga}_{0.7}$ As heterojunction. The interface (see Fig. 1 inset) is separated by undoped $\mathrm{AlGaAs}$ from a bulk doped GaAs cap that serves as an integrated top gate. A $2 \times 2 \mathrm{~mm}^{2}$ square with 16 $\mathrm{NiGeAu}$ Ohmic contacts is fabricated in a field-effect transistor geometry [10]. The Ohmic contacts are selfaligned to the top gate, and carriers are drawn into the 


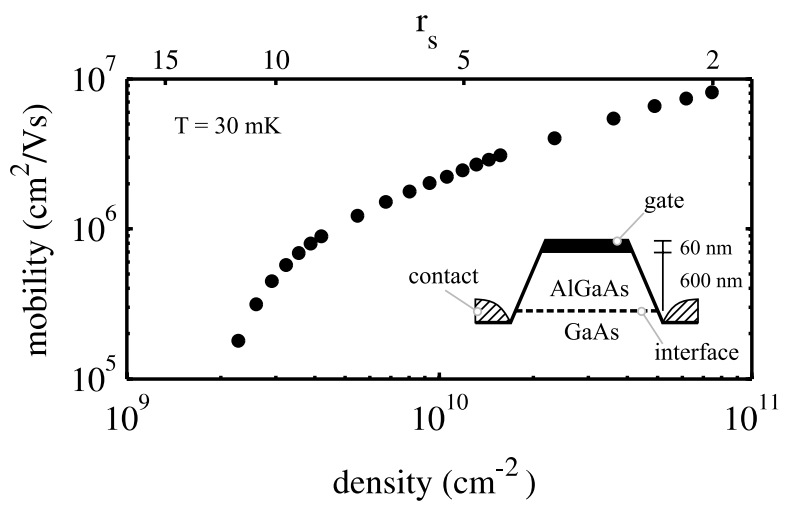

FIG. 1. Plot of mobility as a function of electron density. The top axis indicates $r_{s}$. The inset is a schematic cross section of the undoped heterojunction with self-aligned Ohmic contacts.

channel by applying voltage between the gate and contacts. Once present, the density of the 2DEG is linearly proportional to the gate voltage. A calibration of the density is determined using Shubnikov-de Haas oscillations for a number of gate voltages. Although using undoped heterostructures requires complicated processing, an ultralow density can be achieved without the penalty of a substantial fixed disorder potential arising from remote delta doping.

The mobility, $\mu=1 / n e \rho$, is determined at $T=$ $30 \mathrm{mK}$, where we empirically observe that the resistivity no longer changes with temperature. The mobility is extremely high throughout the entire density range, as is shown in Fig. 1. At the highest density for this sample, the mobility is $8.2 \times 10^{6} \mathrm{~cm}^{2} / \mathrm{V} \mathrm{s}\left(k_{F} \ell=2500\right)$. In other devices that operate at higher density, we have measured a mobility of $1.4 \times 10^{7} \mathrm{~cm}^{2} / \mathrm{V} \mathrm{s}$ at $n=2.5 \times 10^{11} \mathrm{~cm}^{-2}$, a value to which the data in Fig. 1 extrapolate. Since $\mu$ continues to increase at high density, interface roughness scattering is not relevant for data presented here [11].

The temperature dependence of the resistivity for a number of different densities is shown in Fig. 2. The locus of Fermi temperatures is indicated with a dotted line. Three regimes can be identified. First, at high density $\left(n>1.0 \times 10^{10} \mathrm{~cm}^{-2}\right)$ the resistivity is constant at low temperature and then increases as $T$ increases. For intermediate densities $\left(0.29\right.$ to $\left.1.0 \times 10^{10} \mathrm{~cm}^{-2}\right)$, the resistivity is nonmonotonic with temperature. As $T$ increases, $\rho$ is initially constant (see Fig. 4), then increases and decreases to form a local maximum below $T=1 \mathrm{~K}$. The third regime occurs at the lowest densities, with the resistivity decreasing as the temperature increases in the experimentally accessible temperature range.

The phonon contribution to the resistivity at high density is better shown in the inset of Fig. 2, where $\mu^{-1}=$ $n e \rho$ is plotted for $n=0.68$ to $3.36 \times 10^{10} \mathrm{~cm}^{-2}$. The inverse mobility for $T \geq 2 \mathrm{~K}$ is linear, and each density has roughly the same slope of $\sim 3.2 \times 10^{-8} \mathrm{~V} \mathrm{~s} / \mathrm{K} \mathrm{cm}^{2}$ ). Acoustic phonon scattering with both piezoelectric and deformation potential coupling leads to a linear dependence of $\mu^{-1}$ above the Bloch-Grüneisen temperature

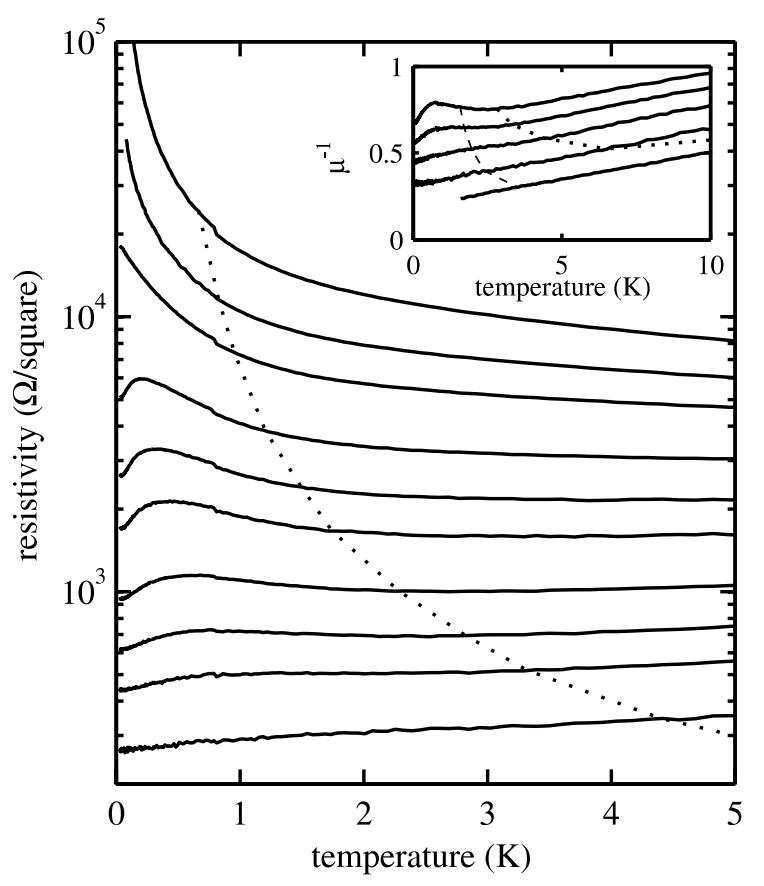

FIG. 2. The main figure shows the resistivity for densities (from the top) $0.16,0.20,0.23,0.29,0.36,0.42,0.55,0.68,0.80$, and $1.06 \times 10^{10} \mathrm{~cm}^{2}$. The inset emphasizes the phonon component of the temperature dependence as straight parallel lines in inverse mobility (in units of $10^{-6} \mathrm{~V} \mathrm{~s} / \mathrm{cm}^{2}$ ) for densities (from the top) $0.68,0.80,1.06,1.57$ and $3.36 \times 10^{10} \mathrm{~cm}^{-2}$. For clarity, the highest two densities in the inset are omitted from the main figure. The dotted line indicates $T_{F}$ and the dashed line in the inset indicates $T_{B G}$. Note the log scale in the main panel and the linear scale in the inset.

( $k T_{B G}=2 k_{F} c \hbar, c$ is the phonon speed of sound). Previously reported experimental [7,8] and theoretical [9] studies of higher density 2DEGs in conventional GaAs heterostructures quantitatively agree with slope found here. This demonstrates that the resistivity of the high density 2DEG is accurately captured using well-known scattering mechanisms. Note that the high temperature resistivity of the low density data in the inset of Fig. 2 remains consistent with phonon scattering even as the nonmonotonic features begin to appear at low temperatures.

As the density is lowered below $n=1 \times 10^{10} \mathrm{~cm}^{-2}$, the resistivity becomes nonmonotonic. A peak in $\rho$ appears for $T<T_{F}$, and the position of the peak shifts to lower temperature for lower densities. The resistivity at the maximum can be up to $23 \%$ larger than the low temperature $(T=30 \mathrm{mK})$ value of the resistivity. The nonmonotonic peak occurs for $T<T_{B G}$, where phonon scattering is strongly suppressed. In this regime of $n$ and $T$, the temperature dependence of ionized impurity scattering must be considered. Screening leads to $\rho(T) \sim T$ for $T \ll T_{F}$ [12], and the crossover from a degenerate Fermi gas to a classical system leads to $\rho(T) \sim 1 / T$ for $T>T_{F}$ [12]. Competition between these effects will lead to a low $T$ resistivity peak. Detailed calculations 
including both ionized impurity and acoustic phonon scattering are presented below (Fig. 5).

For the lowest density that can be attained in our samples $\rho$ decreases monotonically as $T$ increases. While it appears that the 2D electron system is insulating for these densities, comparison to the nonmonotonic regime suggests the possibility that the low $n$ insulating behavior could become nonmonotonic if the electron temperature were lowered further. From the results in Fig. 2, it is clear that a separatrix cannot be identified in this system. To determine the functional form of the insulating regime, low density results were replotted on a $\log -\log$ scale in Fig. 3. The two lowest densities are more consistent with a power law $\left(\rho \sim T^{m}\right)$ dependence than an activated or Mott variable range hopping behavior. For $T<1 \mathrm{~K}, m \approx-0.75$ and -0.5 for $n=0.16$ and $0.20 \times$ $10^{10} \mathrm{~cm}^{-2}$, respectively. While the data are tending toward $1 / T$, as predicted for quantum to classical crossover by scattering theory, both $T<T_{F}$ and $k_{F} \ell<1$ indicate that scattering theory should not be valid at such low density. The large $r_{s}$ values (13.7 and 12.2, respectively) suggest that electron-electron interactions are important. The exact nature of this insulating behavior remains unknown.

In performing $\rho$ measurements at low $n$, great care must be taken to ensure that features do not arise as a result of inhomogeneities in the current flow. The temperature dependence of the resistances is shown in Fig. 4. Two resistances, $R_{A}$ and $R_{B}$, are measured for current flowing, on average, in orthogonal directions. A constant current of $0.3 \mathrm{nA}$ (for the lowest density) to $20 \mathrm{nA}$ (for the highest density) is used in a standard lock-in amplifier measurement at a frequency of $7 \mathrm{~Hz}$. At these currents, transport measurements (e.g., quantum Hall minima) indicate that the electrons cool below $\sim 50 \mathrm{mK}$. The resistivity is determined from the resistances using a procedure prescribed by Van der Pauw [13]. At high density $\left[n=1.57 \times 10^{10} \mathrm{~cm}^{-2}\right.$, Fig. 4(a)], the curves $R_{A}(T)$ and $R_{B}(T)$ almost lie on top of each other, and the temperature dependence is due to phonon scattering. At lower density [Figs. 4(b) and 4(c)], nonmonotonic features appear in both $R_{A}$ and $R_{B}$, but the resistances are no longer identical. Here we observe $R_{A} / R_{B}$ can be as

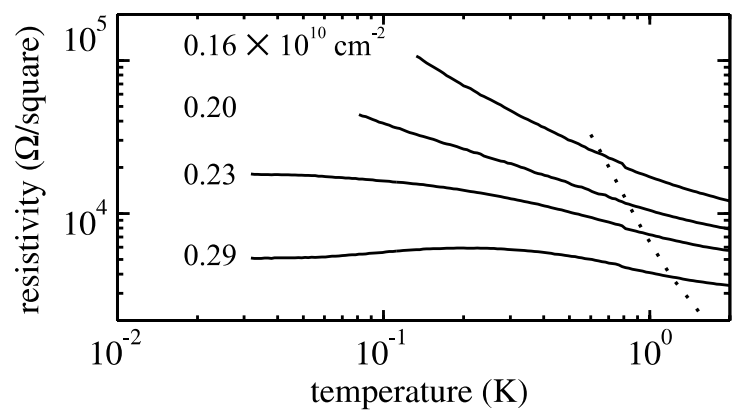

FIG. 3. Resistivity of the lowest densities (noted in figure). $T_{F}$ is indicated by the dotted line. large as 1.6. Earlier designs of undoped samples exhibited a severe ratio; $R_{A} / R_{B}$ approached 30 . This effect originates in the sample fabrication, with the higher resistance always related to the location of the metallic gate contact. We speculate that the gate contact created a locally strained region above the square 2DEG causing inhomogeneous current flow. After redesigning the sample geometry to make the gate contact far from the square measurement region, $R_{A} / R_{B}$ was reduced by more than a factor of 10. While this effect is still visible in Figs. 4(b) and 4 (c), the remaining ratio has a very small $(<10 \%)$ impact on the resistivity [13]. Both $R_{A}$ and $R_{B}$ are nonmonotonic, with resistance peaks located at nearly the same temperature. The qualitative nature of the nonmonotonic resistivity is due to the underlying physics of ionized impurity scattering and not inhomogeneous current flow.

To understand the low temperature $(T<1 \mathrm{~K})$ data, where the phonons contribute little to the resistivity as the system enters the Bloch-Grüneisen regime, we have carried out a microscopic transport calculation using the Boltzmann theory, where we include effects of charged impurity scattering on the electronic resistivity. Our calculation includes the following effects: (i) scattering by ionized charged impurities in the bulk GaAs layer and at the GaAs/AlGaAs interface; (ii) temperature dependent screening, where the electron-charged impurity scattering is taken to be the temperature dependent statically screened Coulomb interaction with the screening calculated within the finite wave vector random phase approximation; (iii) acoustic phonon scattering including

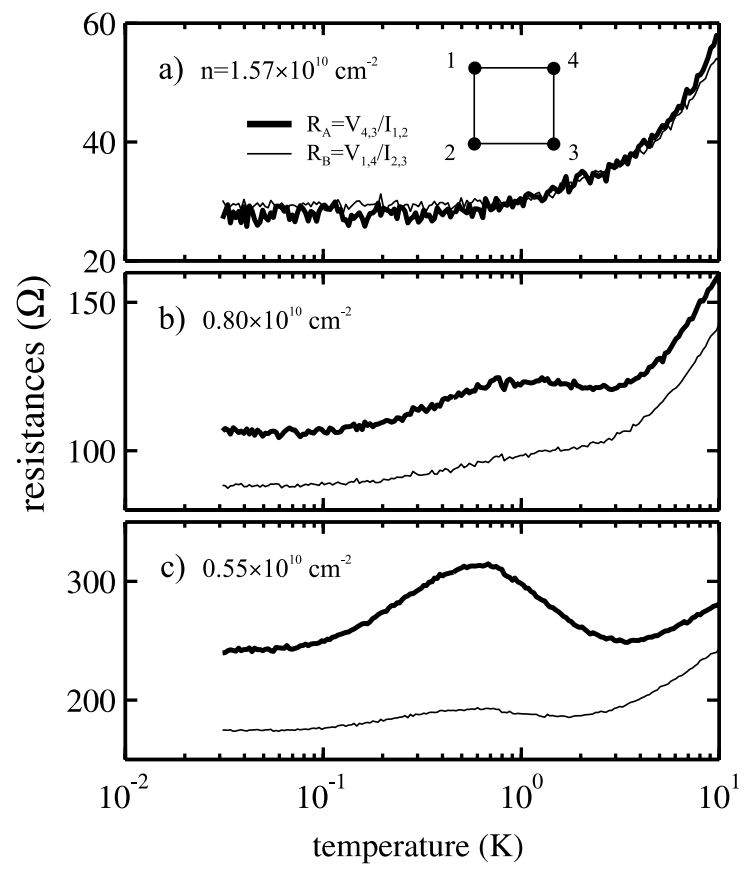

FIG. 4. Four-wire resistances of the corner contacts for densities shown. The diagram labels the contacts. In defining $R_{A}$ and $R_{B}$, the subscripts indicate the current and voltage contacts. 


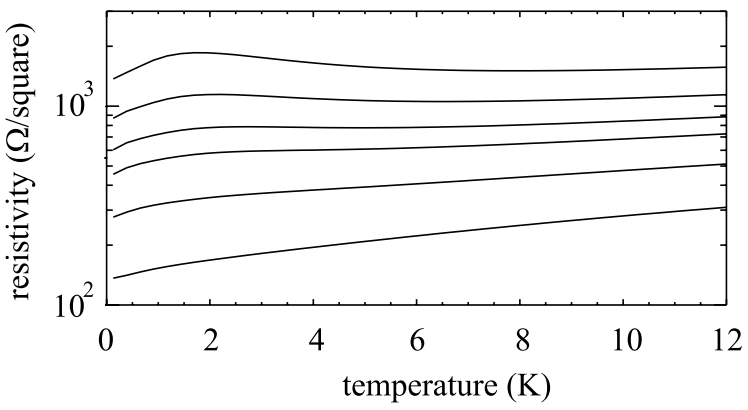

FIG. 5. Results from scattering calculations are remarkably similar to the data in Fig. 3. The density (from the top) is 0.42 , $0.550 .68,0.80,1.06$ and $1.57 \times 10^{10} \mathrm{~cm}^{-2}$. Calculations include acoustic phonons, bulk, and interface impurities.

both deformation potential and piezoelectric coupling; (iv) the quasi-2D nature of the system through subband form factors calculated in the Fang-Howard-Stern variational scheme. This approach is valid for $k_{F} \ell>1$.

We show our calculated results in Fig. 5 for several experimental intermediate and high densities where $k_{F} \ell>1$. Reasonable values are chosen for bulk and interface impurities to allow a comparison of the calculated results to the experimental data shown in Fig. 2. Note that the overall resistivity scale depends on the unknown impurity densities, but the qualitative trends in $\rho(T, n)$ are real and arise from very basic aspects of the underlying scattering mechanisms. The rise in $\rho$ with increasing $T$ at low temperatures is a direct effect of the thermal weakening of screening. The nonmonotonicity of $\rho(T)$ at the intermediate temperatures arises from the competition among temperature dependent screening, quantumclassical crossover, and phonon scattering. The rise at higher $T$ is a phonon effect. Because we are trying to understand the metallic regime, localization effects are not included in this theoretical treatment, and we do not compare to the low density results.

Quantitative understanding requires a more sophisticated theory which includes higher order electronelectron interactions and disorder induced localization corrections. Recently, interaction corrections have been considered by Zala and co-workers [14]. They find that, for a range of temperature, $\hbar / \tau \ll T \ll T_{F}$, where $\tau$ is the transport relaxation time and $\rho(T)$ is expected to be linear. From the slope, the Fermi liquid parameter $F_{\sigma}^{0}$ can be determined. In the data presented here, there is no significant range over which linear $\rho$ is observed for $T \ll$ $T_{F}$ (except for the phonon effect at higher $T$ ), and therefore we do not attempt to determine $F_{\sigma}^{0}$.

In conclusion, we have made resistivity measurements on low disorder gatable 2DEGs over a wide range of densities and temperatures. The resistivity data of high density 2DEGs agree quantitatively with both experimen- tal and theoretical accounts of acoustic phonon scattering in GaAs. For the lowest densities, insulating behavior is observed. At intermediate densities, the resistivity becomes nonmonotonic, with a peak appearing for $T<$ $T_{F}$. At low $T$, the competition between screening $(\sim T)$ and a crossover from a degenerate Fermi system to a classical 2D gas $\left(\rho \sim T^{-1}\right)$ leads to the formation of a peak. Theoretical scattering calculations including both ionized impurities and acoustic phonons in GaAs show excellent qualitative agreement with the nonmonotonic features in the data. This agreement between experiment and theory in the metallic regime suggests that the underlying physics involved is conventional Fermi liquid physics.

We acknowledge outstanding technical assistance from W. Baca and R. Dunn. This work has been supported by the Division of Materials Sciences and Engineering, Office of Basic Energy Sciences, U.S. Department of Energy. Sandia is a multiprogram laboratory operated by Sandia Corporation, a Lockheed Martin Company, for the United States Department of Energy under Contract No. DE-AC04-94AL85000.

[1] S. V. Kravchenko, G. V. Kravchenko, J. E. Furneaux, V. M. Pudalov, and M. D'Iorio, Phys. Rev. B 50, 8039 (1994).

[2] Y. Hanein, U. Meirav, D. Shahar, C. C. Li, D. C. Tsui, and H. Shtrikman, Phys. Rev. Lett. 80, 1288 (1998).

[3] J. Yoon, C. C. Li, D. Shahar, D. C. Tsui, and M. Shayegan, Phys. Rev. Lett. 82, 1744 (1999);H. Noh, M. P. Lilly, D. C. Tsui, J. A. Simmons, L. N. Pfeiffer, and K.W. West, condmat/0206519.

[4] A. P. Mills, Jr., A. P. Ramirez, L. N. Pfeiffer, and K.W. West, Phys. Rev. Lett. 83, 2805 (1999).

[5] Y. Hanein, D. Shahar, J. Yoon, C. C. Li, D. C. Tsui, and H. Shtrikman, Phys. Rev. B 58, 13338 (1998).

[6] C. E. Yasin, M.Y. Simmons, A. R. Hamilton, N. E. Lumpkin, R. G. Clarke, L. N. Pfeiffer, and K.W. West, cont-mat/0204519.

[7] H. L. Stormer, L. N. Pfeiffer, K.W. Baldwin, and K.W. West, Phys. Rev. B 41, 1278 (1990).

[8] J. J. Harris, C. T. Foxon, D. Hilton, J. Hewett, C. Roberts, and S. Auzoux, Surf. Sci. 229, 113 (1990).

[9] T. Kawamura and S. Das Sarma, Phys. Rev. B 42, 3725 (1990);45, 3612 (1992).

[10] B. E. Kane, L. N. Pfeiffer, K.W. West, and C. K. Harnett, Appl. Phys. Lett. 63, 2132 (1993).

[11] J. Heremans, M. B. Santos, K. Hirakawa, and M. Shayegan, J. Appl. Phys. 76, 1980 (1994).

[12] S. Das Sarma and E. H. Hwang, Phys. Rev. Lett. 83, 164 (1999).

[13] L. J. van der Pauw, Philips Res. Rep. 13, 1 (1958).

[14] G. Zala, B. N. Narozhny, and I. L. Aleiner, Phys. Rev. B 64, 214204 (2001). 